Sãdhanā, Vol. 18, Part 6, December 1993, pp. 943-962. (C) Printed in India.

\title{
Vortex simulation of turbulent mixing layers
}

\author{
A J BASU, R NARASIMHA ${ }^{+}$and A PRABHU*
}

National Aerospace Laboratories, Bangalore 560017, India

${ }^{+}$and Jawaharlal Nehru Centre for Advanced Scientific Research, Indian Institute of Science Campus, Bangalore 560012, India

*Department of Aerospace Engineering, Indian Institute of Science, Bangalore 560012, India

\section{MS received 28 September 1992; revised 30 April 1993}

\begin{abstract}
We present here a brief survey of vortex-dynamical simulations of the plane mixing layer using different forms of vortex elements. Points and blobs, which have been widely used in the past, suffer from irregular evolution of the vortex sheet approximating the shear layer, and the rather long time-averaging required to obtain meaningful statistics. A technique recently proposed by the authors, using vortex arcs or sheet elements, has been found to be helpful in avoiding these difficulties. In particular, this technique avoids the singularity in induced velocity associated with point vortex techniques, and is therefore in no need of any form of the "desingularization" that is often introduced in the latter. New results for excited mixing layers using the vortex sheet element model are presented, and are shown to be in good agreement with experimental observations.
\end{abstract}

Keywords. Vortex dynamics; vortex simulation; vortex sheet; mixing layer.

\section{Introduction: Turbulent flows and vortex dynamics}

Turbulent flows have interested scientists and engineers for a long time now, but still remain poorly or marginally understood (see, e.g., Narasimha 1990). Over the last two decades, thc advent of modern vector super-computers and, especially, of parallel computing technology (see Basu et al 1992a in this context), has raised hopes that one might be able to meet the enormous demands made on computing equipment to obtain exact numerical solutions of the governing Navier-Strokes equations describing the evolution of turbulent flows. Such direct numerical simulation of turbulence (resolving all significant scales down to the smallest) has been achieved only for rather moderate Reynolds numbers (of the order of 1000 or so, based on macroscopic velocity and length scales). A direct numerical simulation of the flow past a complete aircraft was estimated to require machines $10^{9}$ times faster and possessing $10^{6}$ times the memory of the most powerful computers available in 1989 (Reynolds 1990). Direct 
numerical simulations are therefore likely to remain limited to modest Reynolds numbers in the foreseeable future, and will perhaps be used mainly to provide new insights into the basic physics of turbulence and guidance in turbulence modelling.

A logical option therefore is to compute exactly the large scales in a turbulent flow, and to model the small scales. Such "large-eddy" simulations could be used as engineering or design tools (Rogallo \& Moin 1984). At an even coarser level, Reynoidsaveraged models are sometimes successful in predicting the mean flow (e.g. Launder 1990), but have serious limitations. The current position (see e.g. Narasimha 1990) is that there are no rational models yet for turbulent flows in general, and those that feed industry are basically ad hoc.

Thus there still exisis a gap in accurate numerical methods for simulating high Reynolds number turbulent flows, of the kind that are of importance to industry. Vortex dynamics offers promise of being an efficient tool for simulating just such flows (see Leonard 1980, 1985 for reviews), although it has its own drawbacks (as we shall discuss below). Its chief advantage is that it provides a conpact Lagrangian method of describing the evolution of 1 rotational flow in terms of vorticity. Now any flow can be thought of as being a combination of potential and rotational parts; as the former can be considered computationally under control, specification of vorticity is virtually equivalent to specification of velocity. Further, as vorticity tends to be concentrated in space, it provides a description of the flow field that is usually compact. Turbulent flows are necessarily rotational or vortical, so it seems an attractive proposition to describe them using vortex dynamics.

Most vortex-dynarnical methods are inviscid, and wouid in principle be eminently suitable for simulating high Reynolds number flows but for certain difficulties. In two-dimensional flow "point" vortices can be used, but they result in irregular roll-up, and are known to be non-convergent as the number of point vortices is increased or the time-step of integration is reduced (Krasny 1986a). (Incidentally, what are referred to as "point" vortices in the plane are actually line vortices extending from $-\infty$ to $+\infty$ in the third dimension perpendicular to the plane of the flow). Moreover, such schemes require large and computationally expensive time-series data for convergence of statistical moments. Vortex "blobs" (i.e. patches of continuously distributed vorticity) have also been used by several workers (e.g. Krasny 1986b). Basu, Prabhu and Narasimha (1992b; hereinafter referred to as BPN) present a method using vortex sheet elements that overcome these difficulties to a large extent. (To be consistent with our terminology of "point" vortices in 2-D flows, these elements would haye to be called vortex "arcs": they are sheets that extend from $-\infty$ to $+\infty$ in the third dimension. The first exploratory calculations using this idea were made by Narayanan 1984 and Mudkavi 1985.) Viscous vortex methods using random-walk are correct only in a statisticai sense (Chorin 1973). Recent work by Fishelov (1990a) on a novel viscous vortex method is promising, but its accuracy still remains to be evaluated for large integration times. There are also various problems in enforcing correct boundary conditions in flows over smooth surfaces (see, e.g. Dutta 1991). Threedimensional methods use vortex filaments (Leonard 1985) and blobs (Fishelov 1990b). In the present paper, we will be concerned mainly with two-dimensional methods using vortex points, blobs and sheets.

We will, in particular, examine two-dimensional vortex-dynamical simulations of the mixing layer, which is a flow that has been studied in great detail in the laboratory. The main reason why the mixing layer has attracted so much attention when compared to other free shear layers is its quasi two-dimensionality, especially under harmonic 
forcing. This characteristic of mixing layers makes them more tractable both analytically and numerically. Thus, reasonable simulations of mixing layers can be made using the tools of two-dimensional vortex dynamics.

The paper is organized as follows. In $\S 2$, we look briefly into the findings from experimental studies of both excited and unexcited mixing layers. In $\S 3$, we review numerical simulations, concentrating mostly on the two-dimensional vortex-dynamical techniques. In $\S 4$, we provide an overview of the vortex sheet element scheme that we have developed and used successfully previously (see BPN). In $\$ 5$, we present some new results for an excited mixing layer using the above scheme. We also briefly describe the previous results for unexcited mixing layer simulation. Section 6 presents some conclusions on the status of vortex dynamical simulations of mixing layers. Details of the present vortex sheet scheme along with some results for unexcited mixing layers have been published elsewhere (BPN), while a direct comparison between the vortex sheet scheme and a point yortex scheme has been reported in Basu et al (1992c). We will draw upon these results whenever necessary.

\section{Experimental observations of the plane mixing layer}

The plane mixing layer forms a subject of fundamental interest in turbulence research (see Spencer \& Jones 1971, Brown \& Roshko 1974, Roshko 1976 and Cantwell 1981). In a typical experimental set-up, two paraliel streains with different velocities $U_{1}$ and $U_{2}$ meet at the end of a finite-length splitter plate, and the mixing region normally extends to a few plate-lengths downstream (figure 1). The turbulent mixing layer is known to grow linearly in the streamwise coordinate $x$, and is conically similar in the variable

$$
\eta=y /\left(x-x_{0}\right)
$$

where $y$ is the coordinate normal to the frec-stream direction, and $x_{0}$ is the $x$-coordinate of a suitable "virtual origin" of the mixing layer, in general different from the trailing edge of the splitter plate. Such similarity is achieved asymptotically at sufficiently large distances downstream, in the limit $\left(x / x_{0}\right) \rightarrow \infty$.

Brown \& Roshixo (1974), through flow-visualization studies, have shown that there is strong spatial coherence in turbulent mixing layers. It has been found that even at very high Reynolds numbers $\left(3 \times 10^{6}\right.$ based on $x$ in the experiments of Dimotakis \& Brown 1976), there are large coherent structures with characteristic length proportional to the thickness of the mixing layer, advecting with the flow and amalgamating nearly

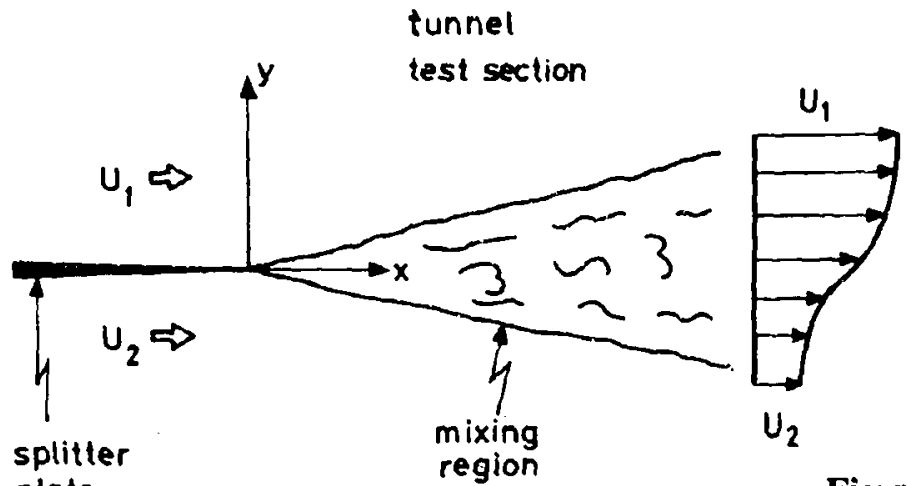

plate

Figure 1. A typical plane mixing layer. 
discontinuously with neighbouring structures at various times. For some distance downstream of the splitter plate the structures are nearly two-dimensional. The dynamics of the mixing layer appears to be governed to a considerable extent by the nearly inviscid interactions of these coherent structures.

It has been observed that the large structures in a mixing layer, and hence its growth, may be controlled significantly by introducing relatively small periodic perturbations in the flow. The effect of such periodic forcing has been studied by Oster \& Wygnanski (1982), Browand \& Ho (1983) and others. A review of forced mixing layer studies may be found in Ho \& Huerre (1984).

The two parameters that significantly affect mixing layer growth are the amplitude $a$ and the frequency $f$ of the forcing. The effect of the frequency is best expressed (see, e.g. Browand \& Ho 1983) in terms of the variation of a non-dimensional thickness $\left(f \theta / U_{c}\right)$ with the non-dimensional streamwise distance $\left(\lambda f x / U_{c}\right)$, where $\theta$ is the local momentum thickness defined as

$$
\theta=\int_{y=-\infty}^{y=+\infty}\left[\left(U_{1}-\bar{U}\right)\left(\bar{U}-U_{2}\right) /\left(U_{1}-U_{2}\right)^{2}\right] \mathrm{d} y,
$$

$U_{c}=\left(U_{1}+U_{2}\right) / 2$ is the advection velocity, $\lambda=\left(U_{1}-U_{2}\right) /\left(U_{1}+U_{2}\right)$ is the nondimensional velocity difference, and $\bar{U}(y)$ is the mean velocity distribution at the streamwise station where $\theta$ is measured.

It is observed that the forced mixing layer may in general be divided into three different "response regimes" as follows (Oster \& Wygnanski 1982).

Regime I: The growth rate may be greatly enhanced (upto twice the unforced value) depending upon the forcing amplitude, over the frequency range $0 \leqslant\left(\lambda f x / U_{c}\right)<1$.

Regime II: In this "frequency-locked" regime (as it is sometimes called), the growth slows down or ceases, and under extremely large forcing amplitudes may even become negative. This regime is usually observed in the frequency range $1 \leqslant\left(\lambda f x / U_{c}\right) \leqslant 2$.

Regime III: The mixing layer resumes growth at nearly the same rate as in the unforced case. This regime lies beyond $\left(\lambda f x / U_{c}\right)=2$.

The effect of the amplitude of forcing is relatively less dramatic. Oster \& Wygnanski (1982) observe that at small amplitudes of forcing (at a given frequency), the growth is enhanced (especially in regimes II and III) due to amalgamation of large structures, whereas at higher amplitudes growth is suppressed in regime II because the flow resonates with the imposed oscillation.

The excited mixing layer has been found to be more nearly two-dimensional in character than the unexcited one, and may therefore be expected to be simulated more accurately by two-dimensional vortex dynamics.

\section{A review of previous vortex dynamical simulations}

The discovery of well-defined quasi two-dimensional coherent structures in the turbulent mixing layer has paved the way for numerical modelling of the flow using twodimensional vortex dynamics (Ashurst 1979; Leonard 1980; Inoue 1985). These efforts 
have been inspired by the hope that a suitable model can be developed for the evolution and roll-up of the vortex sheet that is shed from the end of a splitter plate, the sheet being the result of the tangential velocity discontinuity between the two streams.

\subsection{Temporal simulations}

Long before Brown and Roshko's experiments, there had been attempts to study numerically the temporal evolution of doubly-infinite vortex sheets with periodic boundary conditions due to the Kelvin-Helmholtz instability (Rosenhead 1931; Birkhoff \& Fisher 1959). This configuration has come to be known as the "temporal" mixing layer; it is approached in the limit by a spatial mixing layer when $\left(\Delta U / U_{c}\right) \ll 1$ (where $\Delta U=U_{1}-U_{2}$ ), and can be obtained through a Galilean transformation involving the average free-stream velocity (Corcos \& Sherman 1984). The temporal mixing layer has been experimentally studied by Thorpe (1968).

Since the work of Rosenhead (1931), there have been a large number of numerical attempts to simulate the temporal mixing layer using digital computers (Acton 1976, Krasny 1986 a etc., see table 1). Most authors have discretized the vortex sheet in question by point vortices. Krasny (1986b), following Chorin (1973), has used finitecore vortices or vortex "blobs", which may be seen as part of a technique that essentially removes the induced velocity singularity at a point vortex. While studies using point vortices have reported irregular roll-up (of the interpolating curve representing the vortex sheet) in finite time, the vortex blob method has produced smooth roll-up for small but finite values of a desingularizing parameter. The induced velocity at the discrete vortices in these studies has been computed usually by direct evaluation of the Biot-Savart integral, or by the cloud-in-cell method (see Christiansen 1973). Direct integration is more expensive in terms of computing time but more accurate than the cloud-in-cell method, which cannot resolve flow features smaller than the grid size (see Saffman \& Baker 1979). Reviews of various vortex methods and of numerical studies of the mixing layer may be found in Saffman \& Baker (1979) and Leonard $(1980,1985)$.

Many numerical techniques have been developed and much qualitative insight has been gained into mixing layer flow by these studies. But direct information on the

Table 1. Temporal simulations.

\begin{tabular}{|c|c|c|c|}
\hline Investigator (s) & Method & $\mathrm{Re}$ & Comments \\
\hline $\begin{array}{l}\text { Rosenhead } \\
\text { (1931) }\end{array}$ & $\begin{array}{l}\text { Point } \\
\text { vortex }\end{array}$ & $\infty$ & Showed smooth roll-up at large times \\
\hline $\begin{array}{l}\text { Birkhoff \& } \\
\quad \text { Fisher (1959) }\end{array}$ & $\begin{array}{l}\text { Point } \\
\text { vortex }\end{array}$ & $\infty$ & $\begin{array}{l}\text { Showed that smooth roll-up in Rosenhead's } \\
\text { calculation was due to use of few vortices } \\
\text { and first order time-integration scheme }\end{array}$ \\
\hline $\begin{array}{l}\text { Acton } \\
\quad(1976)\end{array}$ & $\begin{array}{l}\text { Vortex } \\
\text { blobs }\end{array}$ & $\infty$ & Cloud discretization approach used \\
\hline $\begin{array}{l}\text { Krasny } \\
\quad(1986 a)\end{array}$ & $\begin{array}{l}\text { Point } \\
\text { vortex }\end{array}$ & $\infty$ & $\begin{array}{l}\text { Showed that point-vortex scheme does not } \\
\text { converge as number of vortices is increased }\end{array}$ \\
\hline $\begin{array}{l}\text { Krasny } \\
\quad(1986 b)\end{array}$ & $\begin{array}{l}\text { Vortex } \\
\text { blobs }\end{array}$ & $\infty$ & $\begin{array}{l}\text { Showed smooth roll-up at long times. The } \\
\text { desingularizing in the vortex blob scheme } \\
\text { does not have any physical significance }\end{array}$ \\
\hline
\end{tabular}


spatial mixing layer, enabling comparison with experimental data, is not yet available from such computations of the temporal layer.

\subsection{Spatial simulations}

Simulations of the spatial mixing layer flow using vortex dynamical techniques, on the other hand, are far fewer, due to the complexity of the problem and the (consequently) large computational resources required (see table 2). The first such attempt, to our knowledge, was made by Kadomtsev \& Kostomarov (1972). Their method uses point vortices interacting via the Biot-Savart law to simulate the mixing layer; the far downstream is modelled by an absorbing wall. Ashurst (1979) has made a detailed study using vortex blobs. He uses numerical techniques like a long-range cut-off and exponential spreading (in time) of the core of each discrete vortex, and imposes a "random walk" (as in Chorin 1973) to simulate viscous diffusion. Leonard (1980) replaces the upstream splitter plate and the far downstream by semi-infinite vortex sheets; the vortex sheet in the domain of interest is replaced by discrete vortices with Gaussian cores. Inoue (1985) has made an essentially inviscid computation of a mixing layer flow using vortex blobs; a similar methodology has been used by Inoue \& Leonard (1987) in a more recent simulation of both excited and unexcited mixing layers. Ng \& Ghoniem (1985) have simulated shear layers enclosed in a duct. Threedimensional shear layers have been simulated using vortex filaments by Leonard (1985) and Ashurst \& Meiburg (1988), among others; however, we will not pursue three-dimensional simuiations in this paper.

It may be noted that most two-dimensional simulations use discrete vortices to

Table 2. Spatial simulations.

\begin{tabular}{|c|c|c|c|}
\hline Investigator(s) & Method & Re & Comments \\
\hline $\begin{array}{l}\text { Kadomtsev \& } \\
\text { Kostomarov } \\
(1972)\end{array}$ & $\begin{array}{l}\text { Point } \\
\text { vortex }\end{array}$ & $\infty$ & Porous wall used as downstream boundary \\
\hline $\begin{array}{l}\text { Ashurst } \\
\text { (1979) }\end{array}$ & $\begin{array}{l}\text { Vortex } \\
\text { blob }\end{array}$ & 1000 & $\begin{array}{l}\text { Exponential spreading of vortices and } \\
\text { random-walk method used to simulate } \\
\text { viscous diffusion }\end{array}$ \\
\hline $\begin{array}{r}\text { Leonard } \\
(1980)\end{array}$ & $\begin{array}{l}\text { Vortex } \\
\text { blob }\end{array}$ & $\infty$ & $\begin{array}{l}\text { Upstream boundary conditions are taken } \\
\text { into account }\end{array}$ \\
\hline Inoue (1985) & $\begin{array}{l}\text { Vortex } \\
\text { blob }\end{array}$ & $\infty$ & $\begin{array}{l}\text { Upstream and downstream boundary } \\
\text { conditions not satisfied. Computed moments } \\
\text { do not compare well with experimental data }\end{array}$ \\
\hline $\begin{array}{l}\text { Inoue \& } \\
\text { Leonard (1987) }\end{array}$ & $\begin{array}{l}\text { Vortex } \\
\text { blob }\end{array}$ & $\infty$ & $\begin{array}{l}\text { Large number of samples used to obtain } \\
\text { results comparable to experimental data }\end{array}$ \\
\hline $\begin{array}{l}\mathrm{Ng} \& \text { Ghoniem } \\
\text { (1985) }\end{array}$ & $\begin{array}{l}\text { Vortex } \\
\text { blob }\end{array}$ & $\infty$ & $\begin{array}{l}\text { Shear layer in a duct. Results compare well } \\
\text { with experiments }\end{array}$ \\
\hline $\begin{array}{l}\text { Ghoniem \& } \mathrm{Ng} \\
\text { (1986) }\end{array}$ & $\begin{array}{l}\text { Vortex } \\
\text { blob }\end{array}$ & $\infty$ & $\begin{array}{l}\text { Forced shear layer. Upitreara boundary } \\
\text { conditions satisfied properly }\end{array}$ \\
\hline $\begin{array}{c}\text { Basu et al } \\
\text { (1992b) }\end{array}$ & $\begin{array}{l}\text { Vortex } \\
\text { sheet } \\
\text { element }\end{array}$ & $\infty$ & $\begin{array}{l}\text { Both upstream and downstream properly } \\
\text { modelled. Computed moments converge } \\
\text { fast and compare well with experiments }\end{array}$ \\
\hline
\end{tabular}


approximate the vortex sheet in the domain of interest. These attempts follow what is broadly known as the "cloud discretization" approach, where the question of the actual evolution of the vortex sheet (that the discrete vortices are supposed to represent) is considered unimportant in general.

Most of these studies have presented visualizations of the mixing layer by means of streak lines or isovorticity contours, but some have also sought to estimate the growth rate and the profiles of mean longitudinal velocity, Reynolds stresses, and higher order moments. Computed streak line pictures show qualitative similarity with the shadowgraphs of the mixing layer (as in Brown \& Roshko 1974), and thus have strengthened the idea that certain aspects of the large-structure dynamics may be effectively simulated and understood using two-dimensional vortex techniques.

There have been some recent vortex-dynamical attempts to simulate the forced mixing layer (Ghoniem \& $\mathrm{Ng} 1986$; Inoue \& Leonard 1987). These simulations reproduce several characteristic flow-features of the forced mixing layer, including the generation of negative Reynolds stresses in the frequency-locked regime as observed in laboratory studies (e.g. in Oster \& Wygnanski 1982).

\subsection{A critique of iemporal simulations}

Despite the large number of numerical investigations of the mixing layer, the following questions concerning vortex dynamical simulations still remain.

(i) In the limit of infinite Reynolds number, the primary ingredients of the mixing layer flow are infinitesimally thin vortex sheets which cannot be accurately represented by point vortices or vortex blobs, because they cannot reproduce the tangential velocity discontinuity of a vortex sheet faithfully. In particular, point vortices lead to infinite velocities in their immediate neighbourhood, whereas there is no velocity divergence associated with vortex sheets. There has been no report of any smooth solution for a reasonably long time using models of point vortices to approximate a vortex sheet. In fact, the roll-up of the vortex sheet obtained through interpolation in such computations is usually highly irregular, precisely because of the replacement of the sheet by point vortices (Moore 1971): indeed using a larger (but finite) number of point vortices could make matters worse (Krasny 1986a). There are fundamental differences between vortex sheets and an assembly of point vortices: the latter is only a weak solution of the Euler equations, and an assembly of vortex blobs is not an exact solution in any sense, although it may be a reasonable approximation (Saffman \& Baker 1979).

(ii) The caiculation of induced velocity using discrete vortices that are close together is unreliable since they tend to form pairs and rotate around each other. Maskew (1977) reports that the error in calculating the induced velocity at points closer to a vortex than the average vortex spacing may be large.

(iii) Calculations using the cloud discretization approach are physically unrealistic since the question of the actual evolution of the vortex sheet is ignored.

(iv) Calculations using vortex blobs (see, e.g. Krasny 1986b) give smooth roll-up for large times, but the desingularizing method adopted introduces arbitrariness in the form of an additional core-size parameter. Dutta (1989) has shown how an appropriate "size" for vortex blobs can be determined, using as criterion the minimization of the errors that will be committed in certain invariants of two-dimensional vortex dynamics. This method has been applied by him to wake and separated-flow problems, but not 
yet to mixing layers. This technique may provide useful criteria for blob size in the limit of infinite Reynolds numbers.

Drawing inferences about the nature of spatial mixing layer dynamics from the results of temporal mixing layer studies is not always satisfactory; e.g., whereas in a real flow an event like vortex pairing has an effect everywhere (including upstream), in a temporal mixing layer such an event is clearly unable to affect the previous development of the flow. In addition, the spatial layer (unlike the temporal) has no symmetry about any axis.

\subsection{A critique of spatial simulations}

Numerical studies of the spatial mixing layer carried out to date can be subjected to the same criticisms as those listed under (i) to (iv) above regarding the study of the temporal layer, but contain the following additional sources of error as well.

(i) With the notable exception of Leonard (1980), long-range effects of downstream vortices or the downstream boundary condition have not been properly taken into account. Leonard models the downstream as a single semi-infinite sheet, which takes adequate care of the inherent divergence in the problem, but the question of taking into account the lateral spreading of the mixing layer downstream remains.

(ii) The equally important role played by the upstream boundary condition on the splitter plate does not appear to have been recognized except in, e.g. Ghoniem $\& \mathrm{Ng}$ (1986).

(iii) The Kutta condition is usually implemented by shedding a new vortex near the trailing edge of the splitter plate at each time step; Kuwahara (1973) reports that the development of the mixing layer is sensitive to the position where the new vortex is introduced.

It must be admitted that despite many idealizations and incomplete modelling in many vortex dynamical simulations in the past, the mean velocity profiles computed in these simulations compare reasonably well with experimental results. However, the computed maximum Reynolds stresses are often much larger than the experimentally observed values (see BPN). Ashurst matched computation (for a mixing layer at $\mathrm{Re}=$ 1000 based on $x$ ) with experimental results by introducing an exponential spreading of each discrete vortex (as already pointed out, the introduction of such spreading cannot be justified from any solution of the Navier-Stokes equations). The maximum Reynolds stresses (especially the r.m.s. quantity $\mathfrak{u}^{\prime}$ and the Reynolds shear stress $-\overline{u^{\prime} v^{\prime}}$; $u^{\prime}$ and $v^{\prime}$ are the fluctuating components of the streamwise velocity $u$ and normal velocity $v$ respectively) computed by Inoue (1985) using 1000 data-samples are twice the experimental values. A subsequent calculation using a similar model (Inoue \& Leonard 1987) but with about 12,000 data samples, however, brings down the maxima of $\hat{u}^{\prime}$ and $-\overline{u^{\prime} v^{\prime}}$ to the observed order, while yielding a maximum of the r.m.s. quantity $\hat{v}^{\prime}$ that is higher than that previously obtained by Inoue. It was suggested that the differences with experimental results were due to three-dimensionality and viscosity in actual laboratory flows. It may be noted here that one or more of the computed Reynolds stresses may match the experimental results for certain values of the coresize parameter in the vortex-blob approach; this is not unexpected since increasing 
the parameter value will in general result in lower velocity fluctuations in the neighbourhood of a discrete vortex.

It seems evident from the above that there are certain inherent deficiencies in previous models of the spatial mixing layer, especially in the discrete vortex methods used. In particular, the discrepancies in the values of the moments just described could be related to the unrealistically large velocities induced in the neighbourhood of discrete vortices; the intense fluctuations that are a consequence of the approximation do not exist in the limit of a continuous vortex sheet. Hence, it is likely that part of the discrepancies between computed and experimentally observed stresses and moments is due to an over-simplified approach to (rather than to the inherent physical limitations of) two-dimensional vortex dynamics.

\subsection{Previous simulations using vortex sheet elements}

In BPN it has been shown that some of the drawbacks of the vortex point and blob methods mentioned above can be significantly reduced by using a vortex sheet element method, and modelling the upstream and far downstream adequately. In the above method, care had also been taken to satisfy the boundary condition of zero normal velocity on the splitter plate. The results of this study for an unexcited mixing layer show faster convergence for various statistical quantities compared with equivalent point or blob methods, and correspond reasonably well to the available experimental data. In a separate paper (Basu et al 1992c), we have shown, through a direct comparison between a vortex sheet and a point vortex model, that the slower convergence of statistical moments in the point vortex scheme is directly related to higher fluctuations of induced velocities in the neighbourhood of a point vortex.

The vortex sheet element scheme has its drawbacks too. The main problem is to remove a rolled-up "eddy" as it passes out of the main computational domain. In a point vortex scheme, for example, each point vortex is a separate and discrete object, and can be removed independently of others as it moves out of the domain. On the other hand, a vortex sheet is continuous and an element of it cannot be removed independently of others without disturbing the continuity of the sheet. We have developed some techniques such as using a "buffer space" (described in the next section) to tackle this difficulty. We will use this scheme here to compute the evolution of an excited mixing layer, but first briefly describe the vortex sheet element model for both excited and unexcited mixing layers.

\section{The vortex sheet element method}

Following BPN, we study here an idealized form of mixing layer that they call "canonical", namely one that, in the limit of infinite Reynolds number, forms downstream of a semi-infinite splitter plate and extends to infinity far downstream (see figure 2). The advantage of such a configuration is that there are no other external boundaries or parameters, and the divergences associated with the upstream and downstream semi-infinite vortex sheets cancel each other out; thus the problem should be well-posed and possess a well-defined solution. The effects of a finite-length splitterplate and finite computational domain are eliminated in this configuration. 


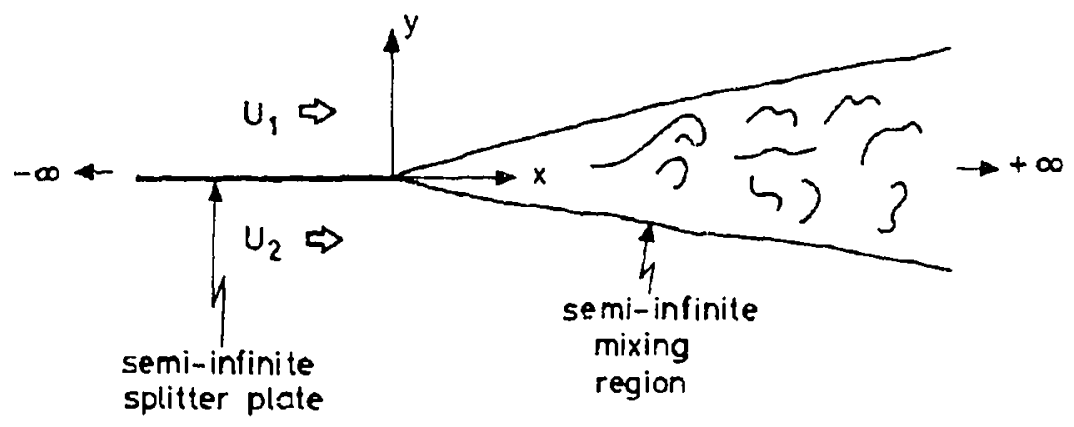

Figure 2. A typical plane canonical mixing layer.

The model and the numerical scheme used, along with an assessment of the accuracy of the scheme, have been discussed in BPN, while the effects (generally small) of various numerical parameters on the computed statistical moments have been reported in Basu et al (1989). Hence we shall describe the scheme here only briefly. We consider a two-dimensional, incompressible canonical mixing layer with free-stream velocities $U_{1}$ above and $U_{2}$ below the splitter plate. In the present computation, viscosity is assumed to be infinitesimal and is present only to generate the necessary vorticity, after which it ceases to play any further role. The flow is modelled in terms of an infinitesimally thin continuous vortex sheet shed from the edge of the splitter plate.

The flow-field is divided into four parts: (i) the region upstream of the trailing edge of the splitter plate, (ii) the computational domain, (iii) a variable "buffer" space and (iv) the downstream far-field (see figure 3). The boundary layer in the upstream region (from $x=-\infty$ to $x=x_{L}$ ) is replaced by a semi-infinite vortex sheet of strength $\Delta U$ $\left(=U_{1}-U_{2}\right)$ per unit length. The doublet sheet used to satisfy the boundary condition of zero normal velocity on the plate is taken to extend only upto a finite distance (say from $x=x_{R D}$ to $x=x_{L D}$; figure 3). Because the boundary condition is more severe near the trailing edge of the plate, we shall use shorter clements there. This is

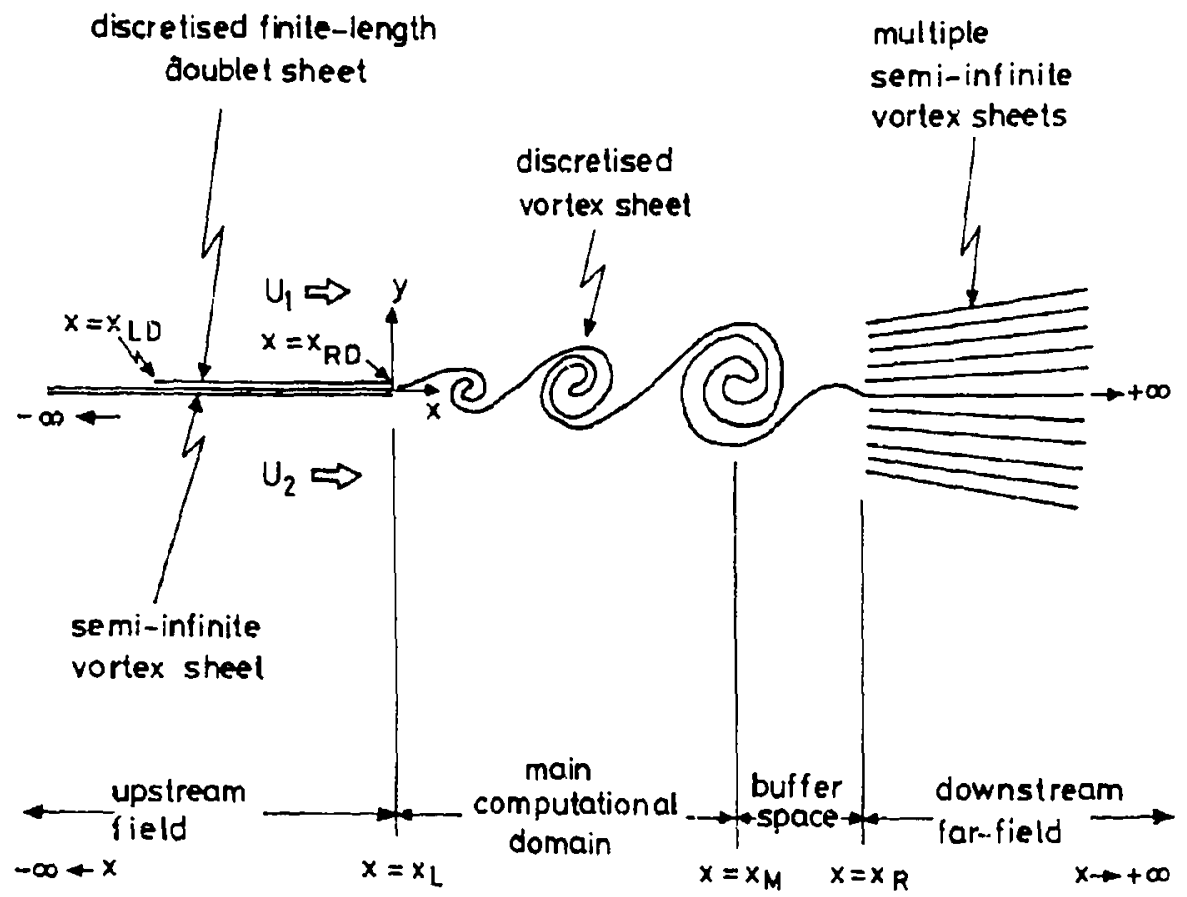

Figure 3. The vortex sheet model of a canonical mixing layer. 
accomplished by dividing the sheet into small linear segments using the scheme

$$
x_{e, i}=x_{R D}-L_{D}\left[(i-1) / N_{D}\right]^{Q_{D}},
$$

where $x_{e, i}$ represents the $x$-coordinate of the right-hand edge of the ith doublet element, $L_{D}$ the total length of the doublet sheet, $N_{D}$ the number of doublet elements and $Q_{D}$ a parameter that controls the element-density near the trailing edge of the splitter plate. Inside the computational domain (extending from $x=x_{I}$ to $x=x_{M}$ ), the vortex sheet is divided into short linear elements of constant strength. A small sinusoidal perturbation of the form

$$
y=A_{0} \sin \left(2 \pi x / W_{0}\right)
$$

(where $A_{0}$ and $W_{0}$ are respectively the amplitude and wavelength of the perturbation) may be given at $t=0$ to accelerate the rolling-up process. The actual values of $A_{0}$ and $W_{0}$ (when $A_{0}$ is sufficiently small) are found not to affect the final state of the mixing layer, at least in terms of the computed statistical moments (Basu et al 1989). At each time-step, the induced velocities at the mid-points of the elements are computed by direct evaluation of the Biot-Savart integral. The velocities at the end-points (where the integral is singular) are then calculated using a linear interpolation scheme. The elements are moved over each time-step using a first order Euler time-integration scheme while maintaining the circulation constant. If an element is seen to stretch beyond twice its initial length, it is divided into halves and the evolution of the new elements is recomputed from the previous time-step.

The Kutta condition is satisfied by shedding an element of strength $\Delta U$ tangential to the splitter plate at each time-step. As each vortical structure leaves the computational domain, it is accommodated in the variable buffer space (extending from $x=x_{M}$ to $x=x_{R}$ ) until the whole structure has left the domain; it is then replaced by the far-field vorticity distribution (to be discussed). The buffer space provides a "soft" coupling between the computational domain and the downstream far-field.

To start with, the downstream far-field (from $x=x_{R}$ to $x=+\infty$ ) is replaced by a semi-infinite vortex sheet of strength $\Delta U$ per unit length. The mean vorticity profile is then computed inside the domain, and the downstream far-field is replaced by a collection of semi-infinite vortex sheets approximating this distribution assuming conical similarity. This iterative procedure is repeated until the profile converges in the domain. All subsequent calculations are made with this "converged" mean vorticity distribution far downstream.

For studies of the excited mixing layer, the flow is forced by sinusoidally varying the normal velocity at the trailing edge of the splitter plate; the incremental velocity is given by

$$
\Delta v\left(x=x_{L}, t\right)=a \sin (2 \pi f t) .
$$

In this case $A_{0}$ is taken to be equal to zero, since perturbation to the vortex sheet is anyway provided through the harmonic forcing described above.

\section{Results}

In this section, we present the results of a numerical simulation of a canonical mixing layer with a non-dimensional velocity difference $\lambda=0.25$. This particular value is 
chosen because of the wealth of laboratory data (Spencer \& Jones 1971, Oster \& Wygnanski 1982 etc.) and numerical results (Inoue 1985, Inoue \& Leonard 1987 etc.) available at this value of $i$, thereby making comparisons easier. The various parameters describing the model for this simulation are:

$$
\begin{aligned}
& U_{1}=2.5, \quad U_{2}=1.5, \quad \Delta t=0.01, \\
& x_{L}=0, \quad x_{M}=2, \quad x_{R} \geqslant 2, \\
& x_{L D}=-10, \quad x_{R D}=0, \quad N_{D}=20, \quad Q_{D}=5 \text {; }
\end{aligned}
$$

$\Delta t$ is the time-step of integration. In addition, the frequency $f$ and the amplitude $a$ of forcing are to be specified for an excited mixing layer. For an unexcited mixing layer, we chose $A_{0}=0.01$ and $W_{0}=0.25$; for the excited layer, $A_{0}=0$.

At time $t=0$, the downstream far-field is approximated by a semi-infinite vortex sheet of strength $y=1$ per unit length, extending from $x=2$ to $x=+\infty$ along the $x$-axis. This distribution is subsequently improved by the iterative technique described previously (see $\S 4$ ), using upto 21 semi-infinite vortex sheets. The computed mean vorticity profile is found to converge satisfactorily within 4 iterations in most cases. Once convergence has been achieved, all subsequent calculations are performed using this "converged" vorticity distribution to provide boundary conditions far downstream.

The present computations are inviscid, and thus have an effective Reynolds number of infinity. However, comparison with experimental data at finite but high Reynolds numbers $\left(10^{5}\right.$ or more) is justified since one can expect statistical similarity among flows at high Reynolds numbers (Townsend 1976). Hence the present simulation should capture the essence of high Reynolds number mixing layers. The calculations being two-dimensional, one can expect some difference with the experimental studies which are three-dimensional. However, computed gross quantities such as the maximum Reynolds stresses should at least be of the observed order. Comparison with published two-dimensional vortex dynamical simulations, on the other hand, should bring out the relative merits and drawbacks of the present method.

\subsection{The unexcited mixing layer}

The unexcited mixing layer has been simulated using the vortex sheet element scheme for the above-mentioned parameters; details are available in BPN. However, we shall briefly summarize the main results below.

The computations have been carried on for 4200 time steps, that is from $t=0$ to $t=42$. Beyond $t=1$, the mixing layer that develops over the computational domain is found to be statistically steady. The vortex sheet in the computational domain rolls up into large vortical structures, in a manner very similar to what is seen in the shadowgraphs of Brown \& Roshko (1974) for plane turbulent mixing layers. The computed mixing layer has been found to grow nearly linearly and the growth rate is similar to that seen in laboratory experiments. The virtual origin has been estimated to be located at $(0.34,0)$ downstream of the end of the splitter plate. Computed invariants such as the Hamiltonian have been found to be well-conserved, thereby confirming the accuracy of the computations.

The computed mean streamwise velocity profile converges when averaged over just 200 data-samples. This is much less than what is required when point vortex schemes are used, for example (see Basu et al 1992c). The mean velocity defect $\left(\bar{U}-U_{2}\right)$ is 


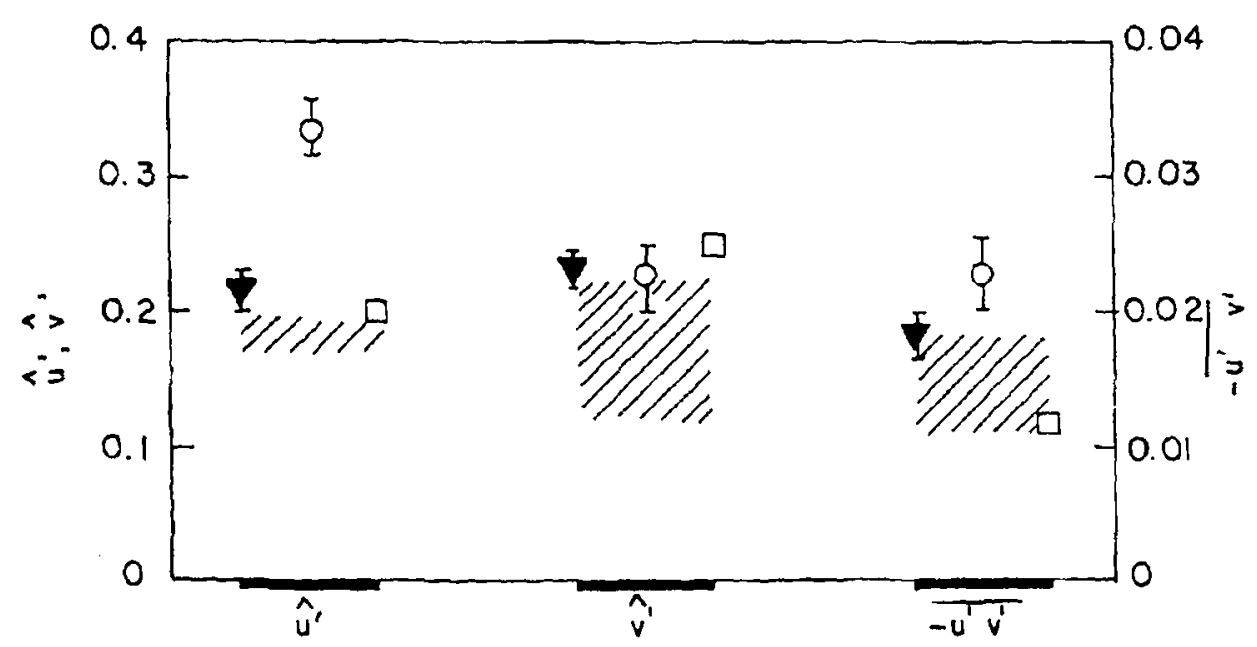

Figure 4. The maxima of r.m.s. $u^{\prime}, \hat{v}^{\prime}$ and the Reynolds stress $-\overline{u^{\prime} v^{\prime}}$ obtained from: $\nabla$, Basu et al (1992b); $O$. Inoue (1985); $\square$, Inoue \& Leonard (1987). The spread of relevant experimental data (from Basu et al 1992b) is shown by hatched lines.

found to be self-similar when plotted against the non-dimensional variable

$$
\eta-\eta_{0}=\left(y-y_{0}\right) /\left(x-x_{0}\right)
$$

here $y_{0}$ refers to the $y$-coordinate where $\bar{U}(y)=U_{c}$. The computed mean velocity profile agrees well with the published experimental data:

The computed mean profiles of r.m.s. turbulence intensities $\hat{u}^{\prime}$ and $\hat{v}^{\prime}$, along with the Reynolds shear stress - $\overline{u^{\prime} v^{\prime}}$, have been found to converge when averaged over only 1000 velocity samples. The computed profiles are found to be similar when plotted against the non-dimensional parameter $\left(\eta-\eta_{0}\right)$. Figure 4 shows a direct comparison between the laboratory data and the computed results obtained by BPN and other vortex-dynamical studies. The maximum values of the stresses computed by the present method fall at the higher end of the scatter in the experimental data. The point to be noted is that similar correspondence with experimental data has been achieved in other vortex dynamical computations (Inoue \& Leonard 1987, for example) only on using about 12,000 data-samples for averaging purposes. Inoue (1985) averages over 1000 data-samples and obtains maximum $\hat{u}^{\prime}$ that is about twice the experimental value. It is clear from this that the vortex sheet element scheme shows faster convergence.

Similar behaviour has been seen while computing the third-order moments $\overline{u^{\prime 3}}$, $\overline{v^{\prime 3}}, \overline{u^{\prime 2} v^{\prime}}$ and $\overline{u^{\prime} v^{\prime 2}}$. Of these, the $\overline{u^{\prime 2} v^{\prime}}$ and $\overline{u^{\prime} v^{\prime 2}}$ profiles converge reasonably well when averaged over 4000 data-samples, but $\overline{u^{\prime 3}}$ and $\overline{v^{\prime 3}}$ are slower in convergence, and need more data-samples than we could afford with the available computational resources. However, the computed moments, especially $\overline{u^{2} v^{\prime}}$ and $\overline{u^{3}}$, showed reasonably close correspondence with experimental data. The details of comparison with experimental and other numerical results are given in BPN. In figure 5, we show laboratory data and the present computed results along with those of Inoue (1985).

Thus we have found that the vortex sheet element method simulates the unforced mixing layer reasonably well, and the computed statistical moments converge faster when compared to other discrete vortex methods. 


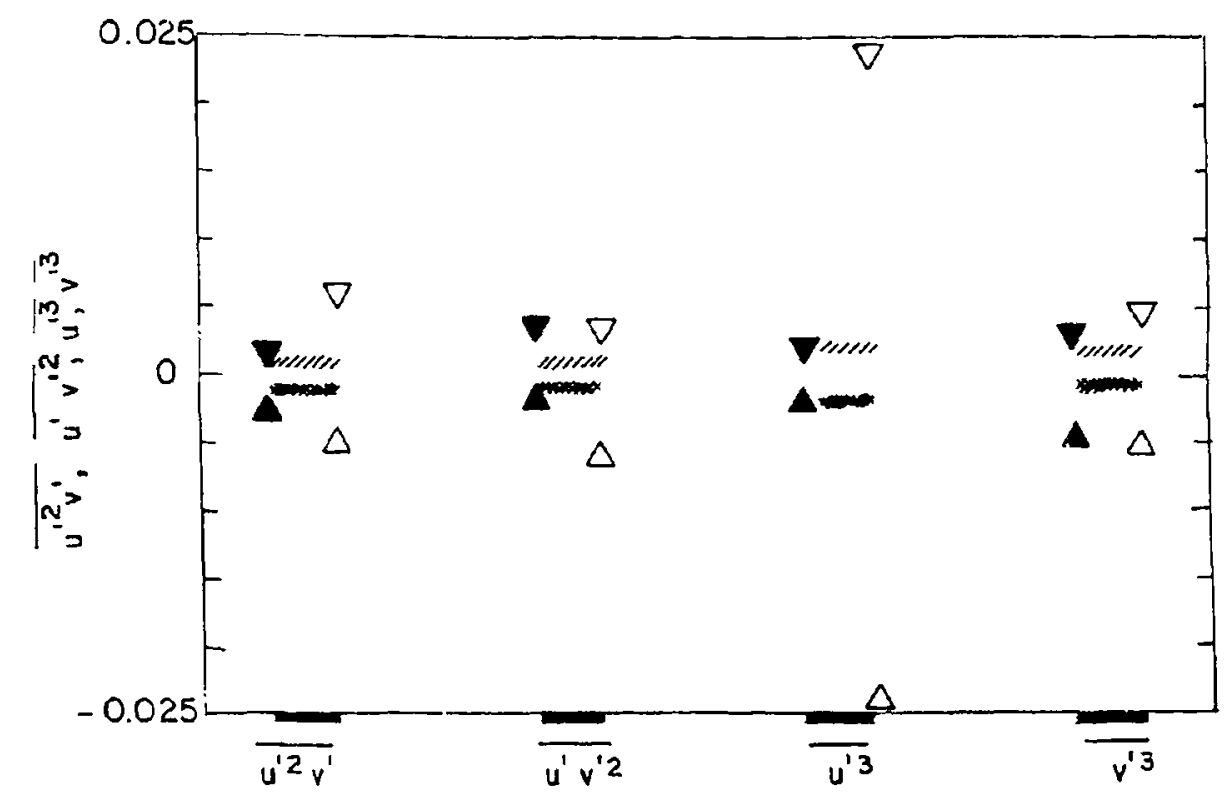

Figure 5. The maximum and minimum values of the third-order moments $\overline{u^{\prime 2} v^{\prime}}$, $\overline{u^{\prime} v^{\prime 2}}, \overline{u^{\prime 3}}$ and $\overline{v^{\prime 3}}$ obtained from: $\nabla$ and $\Delta$, Basu $e t$ al $(1992 b) ; \nabla$ and $\Delta$, Inoue (1985). The spread of the relevant experimental data (Spencer \& Jones 1971) is shown using single-hatched and cross-hatched areas.

\subsection{The excited mixing layer}

In the results presented below, the vortex sheet element method has been used to compute the spatial development of a mixing layer subject to sinusoidal forcing in the normal velocity component, for different forcing frequencies and amplitudes. We will present the effects of forcing frequency and amplitude separately. The results are compared with the experimental data of Oster \& Wygnanski (1982) and Browand \& Ho (1983), and other two-dimensional vortex dynamical simulations such as Inoue \& Leonard (1987).

5.2a Effect of frequency of forcing: Here we shall study the effect of forcing frequency on the development of mixing layers by examining solutions at $f=5,10$, 15 and 20. The amplitude of forcing is kept constant at $a=0.1$ in all cases. The roll-up of the vortex sheet inside the computational domain at time $t=2$ for the four different cases are shown in figure 6 . As may be seen, the computed vortex sheet intersects itself at places, which is of course a violation of the laws of vortex dynamics. But, as we have previously argued (see BPN), these intersections can be removed using shorter elements and smaller time-steps, but at the expense of higher computational cost. However, while such intersections are not physically or mathematically correct, their effect on the mean flow quantities is negligible, and the additional computing effort required to remove them is unnecessary if interest is confined to certain mean quantities. It must be recalled here that in point vortex methods, merely increasing the number of vortices does not guarantee convergence (Krasny 1986a).

There is clear evidence of the pairing process for low-frequency forcing at $f=5$ (figure 6). The frequency-locked regime, if one exists, is perhaps outside the computational domain in this case. As we increase the forcing frequency, the frequency-locked regime may be observed inside the domain, though its length gets progressively shorter. 

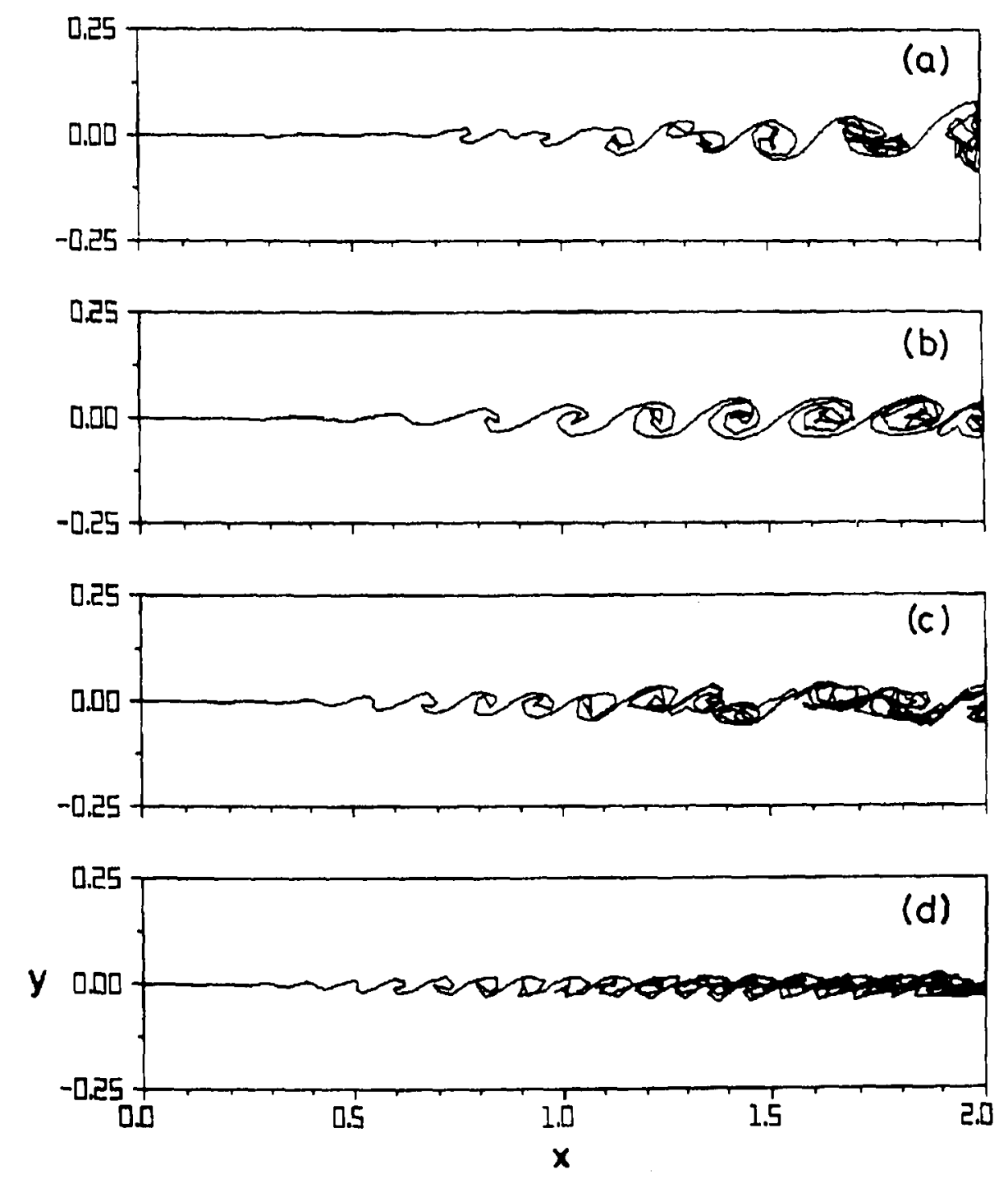

Figure 6. Effect of frequency of forcing: roll-up of the vortex sheet at time $t=2$ due to: $f \geq 5$ (a), 10 (b), 15 (c), and 20 (d); for all cases $a=0.1$.

For $f=15$, there is pairing and renewed growth downstream of the frequency-locked regime, whereas for $f=20$, there does not seem to be any enhancement of the mixing layer growth inside the computational domain, even though many of the structures downstream appear to be in a state of partial amalgamation through mutual interaction. The qualitative scenario of the effect of forcing frequency on mixing layer growth described so far is essentially similar to that found in other experimental (e.g. Oster \& Wygnanski 1982) and numerical (Inoue \& Leonard 1987) studies.

In figure 7, we present the computed variation of the local momentum thickness $\theta$ along $x$ as a function of the forcing frequency (figure 7 may be compared with figure 13 of Oster \& Wygnanski 1982, and figure 8a of Inoue \& Leonard 1987; these results are qualitatively similar to the present one and are not superposed on figure 7 since that would spoil the clarity of the figure). The unforced mixing layer growth from BPN is shown superposed for comparison. We can clearly see evidence of frequencylocked regimes from this figure, especially for $f=10,15$ and 20 .

The effect of forcing frequency is better expressed if $x$ and $\theta$ are non-dimensionalized as $\lambda x f / U_{c}$ and $\theta f / U_{c}$, respectively. The results are shown in figure 8 . The asymptotic 


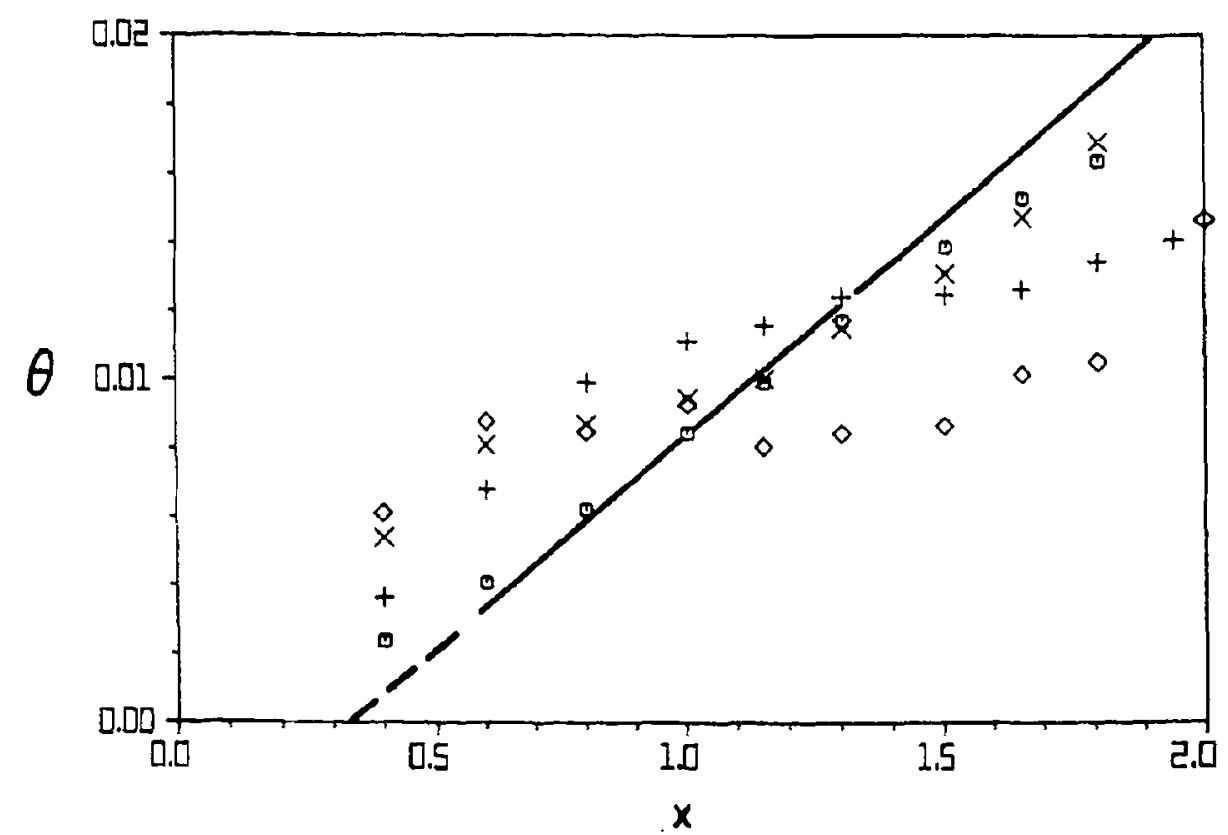

Figure 7. Vorticity thickness growth of computed mixing layer shown as a function of the forcing frequency: $f=5(\square), 10(+), 15(x)$, and $20(\diamond)$; for all cases $a=0 \cdot 1$. The solid line shows the unforced mixing layer growth obtained by Basu et al (1992b).

growth rate as in BPN is superposed along with the growth law for the forced mixing layer (Browand \& Ho 1983). We see that for $f=5$, the frequency-locked regime which appears between $1<\lambda f x / U_{c}<2$ is obviously outside the computational domain as mentioned before. For $f=10$ and 15 , the computed results follow the growth law

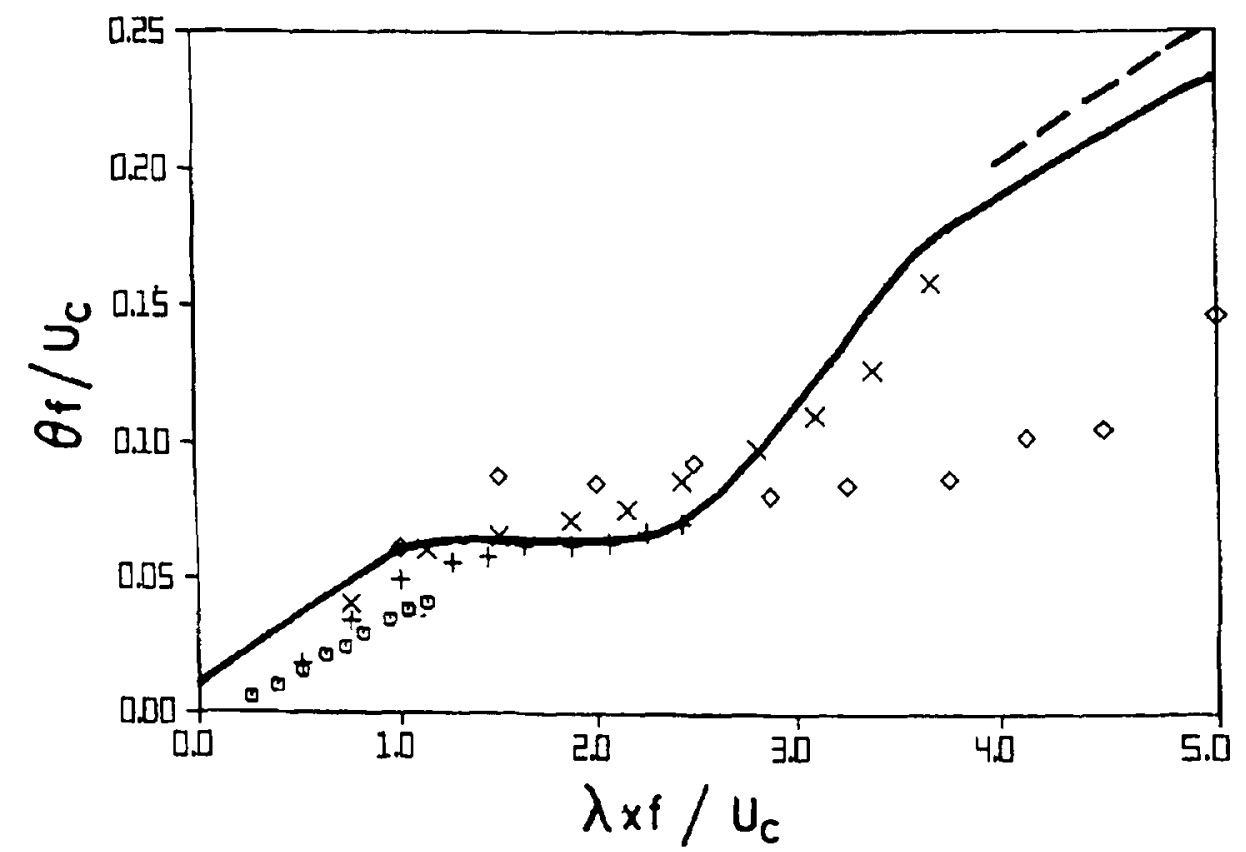

Figure 8. Effect of frequency of forcing: scaled growth of computed mixing layer for: $f=5(\square), 10(+), 15(x)$, and $20(\diamond)$; for all cases $a=0 \cdot 1$. The asymptotic growth rate of the unforced mixing layer as computed by Basu et al (1992b) is shown as a dashed line, along with the growth law for the forced mixing layer (Browand \& Ho 1983) as a solid line. 
curve quite well, whereas for $f=20$, the growth rate in regime III falls much below the predicted one. This behaviour can actually be noticed also in the results of Inoue and Leonard, who point out that Oster and Wygnanski use forcing frequencies that are much smaller than the predominant frequency found at the end of the splitter plate in an unexcited mixing layer. The growth law suggested by Browand and Ho has been based on the data of Oster and Wygnanski, and hence fails to take into account the effects of forcing frequencies that are high compared to the predominant frequency. Based on these results, it appears that the growth law of Browand and Ho is valid only for forcing frequencies smaller than the predominant frequency. At high forcing frequencies, the growth in regime III (at least upto $\lambda x f / U_{c}=4.5$ ) is very much below the predicted rate. The present results are thus qualitatively comparable to both experimental data and other numerical results, but show rapid convergence.

$5.2 \mathrm{~b}$ Effect of amplitude of forcing: We shall examine here the effect of four different amplitudes of forcing, $a=0.01,0.05,0.1$ and 0.5 , kecping the forcing frequency constant at $f=10$. These forcing amplitudes cover a wide range, going up to the order of the
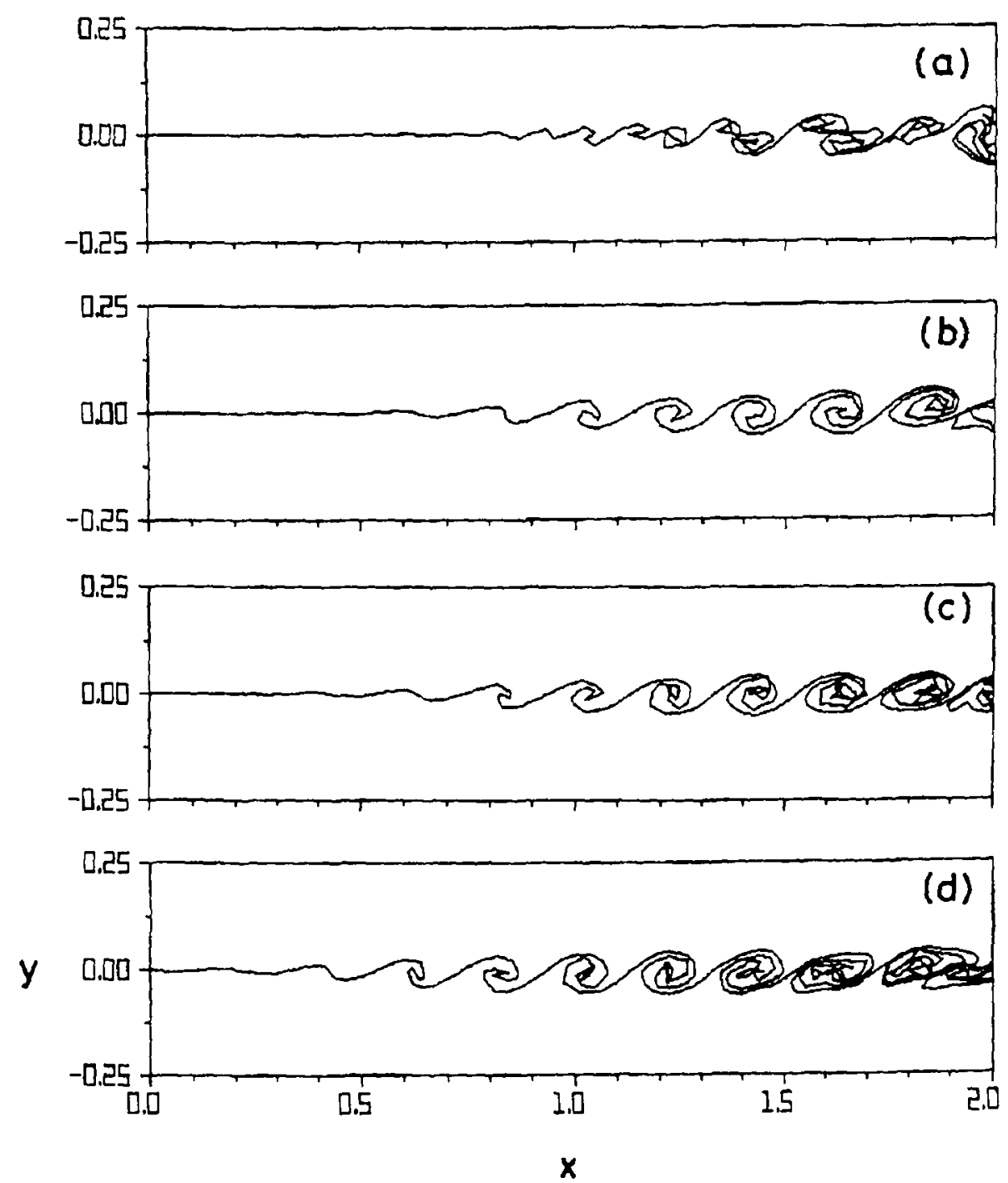

Figure 9. Effect of amplitude of forcing: roll-up of the vortex sheet at time $t=2$ due to: $a=0.01(\mathbf{a}), 0.05$ (b), 0.1 (c), and 0.5 (d); for all cases $f=10$. 
normal velocity fluctuations in the unforced mixing layer. It may be noted that the amplitude here refers to that of the normal velocity perturbation at the trailing edge of the splitter plate, and not (as in the experiments of Oster and Wygnanski) to the displacement of a trailing edge flap. Because of this, quantitative comparison with experiments is difficult.

Figure 9 shows the roll-up of the vortex sheet at time $t=2$ for different forcing amplitudes. With increasing amplitude of excitation, the mixing layer starts evolving closer to the trailing edge of the splitter plate, and the three different response regimes may be observed inside the domain for $a=0.1$ and 0.5 . For low-amplitude forcing ( $a=0.01$ ), amalgamation through pairing (as also observed by Oster and Wygnanski) is seen. At higher amplitudes of forcing the linear downstream growth of the mixing layer appears retarded; there is some indication that it becomes negative for $a=0.5$.

The mixing layer growth in terms of the momentum thickness $\theta$ at various $x$-stations is shown in figure 10 as a function of $a$; unforced mixing layer growth from BPN is superposed for comparison (figure 10 may be compared with figure 12 of Oster \& Wygnanski 1982 and figure 12a of Inoue \& Leonard 1987; these results are qualitatively similar to the present one and are not superposed for the sake of clarity in presentation). For $a=0.01$, the initial growth rate is nearly the same as in the unforced case. For $a=0.05$, we can see the beginning of a frequency-locked regime inside the computational domain, whereas for $a=0.1$ and 0.5 regime III also appears inside the domain. The growth rate for $a=0.1$ is higher than that for the unforced case. For $a=0.5$, there is indeed a negative growth rate region around $x=1 \cdot 2$. The above scenario is in qualitative agreement with previous experimental and numerical results. Oster and Wygnanski provide data only for a short distance downstream of the splitter plate $\left(\lambda x f / U_{c}=1 \cdot 6\right)$, so it is not possible to compare the present results (upto $2 \cdot 5$ ) with experimental data for large downstream distances.

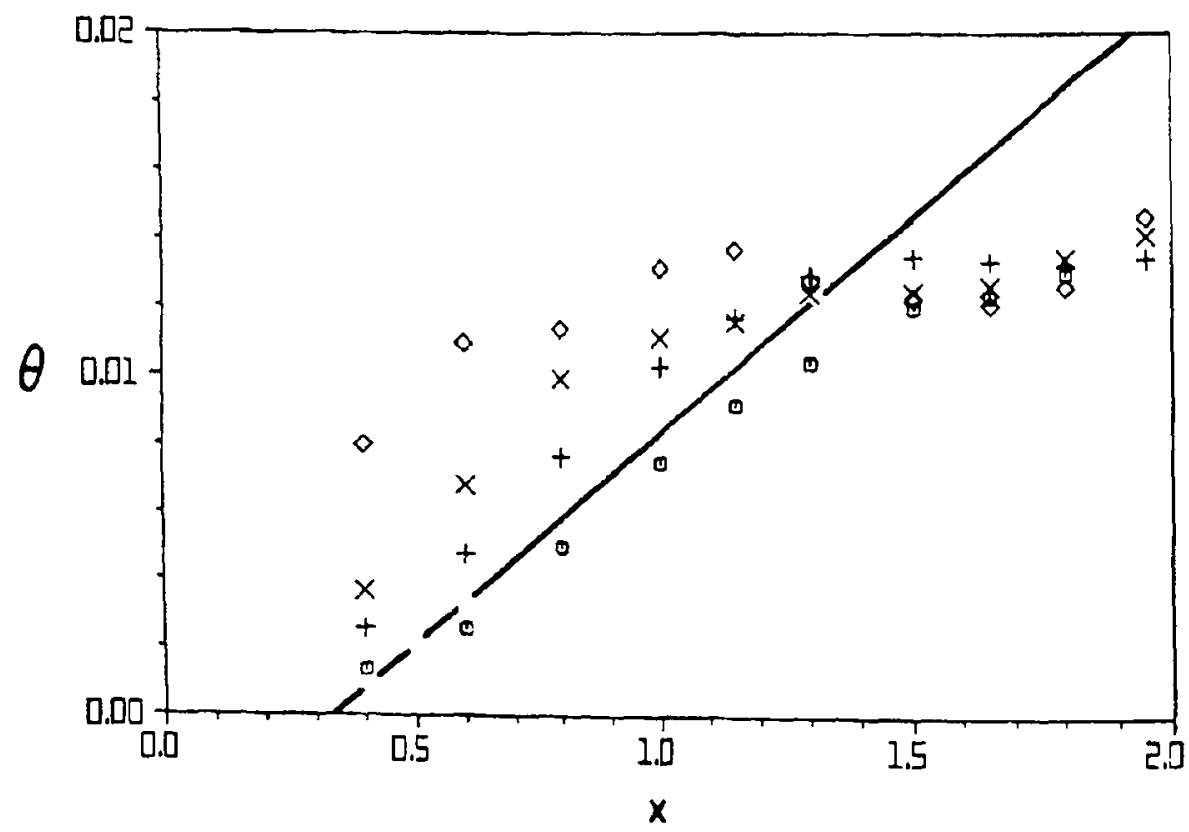

Figure 10. Vorticity thickness growth of computed mixing layer shown as a function of the forcing amplitude. $a=0.01(\square), 0.05(+), 0.1(x)$, and $0.5(0)$; for all cases $f=10$. The solid line shows the unforced mixing layer growth obtained by Basu et al (1992b). 


\section{Conclusions}

In this paper, we have reviewed the status of two-dimensional vortex dynamical simulations of both excited and unexcited mixing layers. Most vortex dynamical simulations use vortex points and blobs to approximate the vortex sheet that makes up the shear layer at high Reynolds numbers. Such methods have been found to have two main drawbacks: (i) irregular evolution of the vortex sheet in question; (ii) long averaging time required for convergence of statistical moments. In addition, most simulations carried out to-date do not handle the upstream and downstream boundary conditions satisfactorily. The present vortex sheet element method, on the other hand, has been found not to suffer from the above mentioned difficulties. The differences observed between the results from the point and sheet schemes can be traced to the large fluctuations in the velocity induced in the neighbourhood of vortex points. Such fluctuations are noticeably small in the vortex sheet element method.

The vortex sheet element method simulates the features of both excited and unexcited mixing layers reasonably well. The main practical advantage of the method is its rapid convergence in computing mean stresses and moments. This method can easily be extended to 3-D as well, using vortex panels instead of 2-D vortex sheet elements.

This work has been partially supported by the Department of Science and Technology, Government of India. One of the authors, AJB, gratefully acknowledges support from the Jawaharlal Nehru Centre for Advanced Scientific Research during the latter part of this study. Thanks are also due to the students and staff of the Centre for Atmospheric Sciences, Indian Institute of Science, for their help and cooperation, and for letting us use their computational facility.

\section{References}

Acton E 1976 The modelling of large eddies in a two-dimensional shear layer. J. Fluid Mech. 76: $561-592$

Ashurst W T 1979 Numerical simulation of turbulent mixing layers via vortex dynamics. Turbulent shear flows (eds) F Durst, B E Launder, F W Schmidt, J H Whitelaw (New York: Springer-Verlag) 1: 402-413

Ashurst W T, Meiburg E 1988 Three-dimensional shear layers via vortex dynamics. J. Fluid Mech. 189: 87-116

Basu A J, Narasimha R, Prabhu A 1989 Numerical simulation of a plane "canonical" mixing layer using a vortex sheet model. Report No. 89 FM 1, Aerospace Engineering Dept., Indian Institute of Science, Bangalore

Basu A J, Narasimha R, Sinha U N 1992a Direct numerical simulation of the initial evolution of a turbulent axisymmetric wake. Curr. Sci. 63: 734-740

Basu A J, Prabhu A, Narasimha R 1992b Vortex sheet simulation of a plane "canonical" mixing layer. Comput. Fluids 21(1): 1-30

Basu A J, Prabhu A, Narasimha R $1992 \mathrm{c}$ Modelling plane mixing layers using vortex points and sheets. Applied Mathematical Modelling (submitted)

Birkhoff G, Fisher J 1959 Do vortex sheets roll up? Rendiconti Circolo Mathematico di Palermo Ser. 2. 8: 77-90

Browand F E, Ho C M 1983 The mixing layer: an example of quasi two-dimensional turbulence. Journal de Mecanique Theorique et Applique. Numero Special: 99-120

Brown G L, Roshko A 1974 On density effects and large structure in turbulent mixing layers. J. Fiuid Mech. 64: 775-816

Cantwell B J 1981 Organised motion in turbulent shear flow. Annu. Rev. Fluid Mech. 13:457-515

Chorin A J 1973 Numerical study of slightly viscous flow. J. Fluid Mech. 57: 785-796 
Christiansen J P 1973 Numerical simulation of hydrodynamics by the method of point vortices. J. Comput. Phys. 13: 363-379

Corcos G M, Sherman F S 1984 The mixing layer: deterministic models of a turbulent flow. Part 1. Introduction and the two-dimensional flow. J. Fluid Mech. 139: 29-65

Dimotakis P E, Brown G L 1976 The mixing layer at high Reynolds number: large structure dynamics and entrainment. J. Fluid Mech. 78: 535-560

Dutta P K 1989 Discrete vortex simulation of high Reynolds number flows. Report No. PD CF 8944, National Aeronautical Lab., Bangalore

Dutta P K 1991 Representation of solid boundaries in discrete vortex methods. Report No. TM CF 9102, National Aeronautical Laboratory, Bangalore

Fishelov D 1990a A new vortex scheme for viscous flows. J. Comput. Phys. 86: 211-224

Fishelov D 1990 b Vortex methods for slightly viscous three-dimensional flow. SIAM J. Sci. Stat. Comput. 11: $399-424$

Ghoniem A F, Ng K K 1986 Effect of harmonic modulation on rates of entrainment in a confined shear layer. AIAA paper No. 86-0056

Ho C M, Huerre P 1984 Perturbed free shear layers. Annu. Rev. Fluid Mech. 16: 365-424

Inoue $\mathrm{O} 1985$ Vortex simulation of a turbulent mixing layer. AIAA J. 23: 367-373

Inoue $\mathrm{O}$, Leonard $\mathrm{A} 1987$ Vortex simulation of forced/unforced mixing layers. AIAA paper No. 87-0288

Kadomtsev B B, Kostomarov D P 1972 Turbulent mixing layer in an ideal two-dimensional fluid. Phys. Fluids 15: 1-3

Krasny R 1986a A study of singularity formation in a vortex sheet by point-vortex approximation. J. Fluid Mech. 167: 65-93

Krasny R 1986b Desingularisation of periodic vortex sheet roll-up. J. Comput. Phys. 65: 292-313

Kuwahara K 1973 Numerical study of flow past an inclined flat plate by an inviscid model. J. Phys. Soc. Jpn. 35: 1545-1551

Launder B E 1990 Phenomenological modelling: present and future? In Whither turbulence? Turbulence at the crossroads (ed.) J L Lumley (Heidelberg: Springer-Verlag)

Leonard A 1980 Vortex methods for flow simulation. J. Comput. Phys. 37: 289-335

Leonard A 1985 Computing three-dimensional incompressible flows with vortex elements. Annu. Rev. Fluid Mech. 17: 523-559

Maskew B 1977 Subvortex technique for the close approach to a discretised vortex sheet. $J$. Aircroft 14: 188-193

Moore D W 1971 The discrete vortex approximation of a vortex sheet. Report No. AFOSR1084-69, California Institute of Technology

Mudkavi. V Y 1985 Vortex sheet modelling of a plane turbulent mixing layer. M E thesis, Indian Institute of Science, Bangalore

Narasimha R 1990 The utility and drawbacks of traditional approaches. In Whither turbulence? Turbulence at the crossroads (ed.) J L Lumley (Heidelberg: Springer-Verlag)

Narayanan O 1984 Towards an exact simulation of an inviscid mixing layer. M E thesis, Indian Institute of Science, Bangalore

$\mathrm{Ng} \mathrm{K} \mathrm{K,} \mathrm{Ghoniem} \mathrm{A} \mathrm{F} 1985$ Numerical simulation of a confined shear layer. 10th International Colloquim on the Dynamics of Explosions and Reactive Systems, Berkeley, CA

Oster D, Wygnanski I 1982 The forced mixing layer between parallel streams. J. Fluid Mech. 123: $91-130$

Reynolds W C 1990 The potential and limitations of direct and large eddy simulations. In Whither turbulence? Turbulence at the crossroads (ed.) J L Lumley (Heidelberg: SpringerVerlag)

Rogallo R S, Moin P 1984 Numerical simulation of turbulent flows. Annu. Rev. Fluid Mech. 16: $99-137$

Rosenhead L 1931 The formation of vortices from a surface of discontinuity. Proc. $R$. Soc. London. A134: 170-192

Roshko A 1976 Structure of turbulent shear flows: a new look. AIAA J. 14: 1349--1357

Saffman P G, Baker G R 1979 Vortex interactions. Annu. Rev. Fluid Mech. 11: 95-122

Spencer B W, Jones B G 1971 Statistical investigation of pressure fields in the turbulent twostream mixing layer. AIAA paper No. 71-613

Thorpe S A 1968 A method of producing a shear flow in a stratified fluid. J. Fluid Mech. 32: 693-704

Townsend A A 1976 The structure of turbulent shear flow 2nd edn (Cambridge: University Press) 